\title{
A Testbed for Experiments with Concurrency Control Primitives in Persistent Systems
}

\author{
Michael Flanagan, Alan Fekete, Frans Henskens and John Rosenberg \\ Basser Department of Computer Science, F09, \\ University of Sydney, NSW, 2006, Australia. \\ email: \{ groo, fekete, frans, johnr \} @cs.su.oz.au \\ Telephone: +61-2-692-3423 Fax: +61-2-692-3838
}

In current research operating systems, support for objects and the persistence of these objects has been recognised as an important consideration. Research systems provide support for these concepts in the kernel [4] [7] [3]. In these systems a great deal off effort has gone into integrating persistence and object structures with the other low level functions of the operating system. The low level nature of the support ensures that these concepts are in every respect a seamless part of the operating system which will interact sensibly with other components of the system. There has been a tendency in such systems to ignore the issues of concurrency control and resilience, with the attitude that these concepts are well understood by the database community, and as such, can be easily added on top of the system at a later date.

This paper describes a research project which aims to investigate the implementation of concurrency control and resilience support at the lowest level of the operating system. Concurrency control and resilience are considered to be sufficiently important and complex issues that they merit support at a low layer of the operating system in the same sense that support for persistence belongs at this level. It is a motivating belief for this project that support for fully fledged concurrency control systems in a truly seamless fashion will require appropriate low level functionality provided by the system. The goal of this project is the identification a set of low level operating system primitives through which a variety of both traditional and more advanced concurrency control methods can be implemented on a persistent store. This goal is being approached through the development of a prototype persistent store which will be used as both an experimental environment and a testbed for the demonstration of the viability of the projects results.

\section{Background}

This section describes some essential background. A brief explanation of persistent systems and database concurrency control methodology is presented to give context to the discussion of the motivations and goals of the project, which will be presented in the next section.

\section{Persistent Systems}

"Persistent Systems" describes a class of computing environments which provide a simpler and more coherent data model through all levels of the system [1]. The study of persistent systems extends through all levels of an environment, from low level operating system concepts such as virtual memory and disk management, through to language and user interface designs.

Persistent systems provide environments which remove the distinctions which are traditionally made between classes of data based on the life-expectancy of that data. In a persistent system the user (programmer) accesses a single representation of a data item in a uniform manner irrespective of whether that data is transient data in memory or data which has a lifetime longer than the current process.

In a traditional system transient data stored in memory can represent complex structures with complex graphs of references between structures. In such systems, persistent data must be explicitly moved to stable storage, and must usually be "flattened" to a much simpler representation without explicit references. In persistent systems, however, there is no simple correspondence between the lifetime of a data item and the manner in which it is accessed. Persistent systems allow a uniformity of access for data which exists only for the life of a single process invocation and data which outlives a single process. Both 
transient and persistent data can be in the form of complex data structures with references to other structures.

If data is to be accessed in a uniform manner some method must be used to determine which data is persistent and which data is transient. This is usually done by implementing "Persistence by Reachability." In this model there are some data items which are predetermined or explicitly indicated to be persistent (called persistent roots), and the system causes a data item to "persist" by making it reachable through some chain of references from a persistent root.

\section{Concurrency Control}

Database research has for many years concerned itself with issues related to allowing multiple users to access a common pool of data without adversely affecting each other. This management of simultaneous access to common data by multiple users is called "Concurrency Control" and has been the subject of much investigation and formalisation [5]. This section gives a brief motivation for the need for some method of controlling concurrent accesses and gives an introduction to some of the techniques which have been developed.

The need for controlled concurrent access to data can be demonstrated with a simple example of two financial actions. Consider two threads of control which both wish to add an amount to the same bank account. These two additions can be represented by the following code actions:

DEPOSIT 1
1.1) read value of account 123 into BALANCE1
1.2) add \$10 to BALANCE1
1.3) write BALANCE1 as value of account 123

If these actions are interleaved during a concurrent execution, in the order $(1.1,2.1,2.2,1.2,1.3,2.3)$, the net result will be the addition of only $\$ 20$ to the account.

To avoid problems of interleaving, traditional database techniques use a concept of transactions which embody the principles of Isolation, Atomicity and Resilience. In a system supporting some kind of transaction model simple actions can be grouped together into transactions. The system guarantees a number of properties for transactions. Firstly, if all transactions individually satisfy some consistency constraint on the database (e.g. the total in any account $=$ total credits - total debits) then this constraint will be maintained during concurrent execution of such transactions (Isolation). Secondly a transaction will appear to either entirely complete or have no effect at all (Atomicity). Finally, if a transaction returns an indication that it has successfully completed (committed) then the results of that transaction will not disappear, even in the event of a system crash (Resilience).

Methods of concurrency control include traditional techniques such as Two Phase Locking or Multiversion Timestamp Ordering which provide transactional semantics with strict serializability. Serializability refers to a property of concurrent transaction execution which ensures that the result of a concurrent execution of a collection of transactions will be equivalent to having executed them sequentially in some order. Two Phase Locking ensures this by locking data items to prevent transactions from interfering with each other in a way which would violate the serializability requirement. Multiversion Timestamp Ordering keeps different versions of each object which are labelled with timestamps indicating at what time each version was written. The system then ensures that transactions read appropriate versions of objects to ensure a serializable execution, or force transactions to fail if there is no version which will allow the serializability requirement to be satisfied.

In addition to the traditional methods of concurrency control, some systems have been developed to overcome the shortcomings of the simpler traditional methods. These include systems such as "Nested Transaction" systems. Nested transactions allow subtransactions within a parent transaction. This makes it possible for the system to execute more actions concurrently and allow for more flexibility in the case of error conditions. Rather than the traditional option of aborting all work and starting again, just the subtransaction in which the error

2.1) read value of account 123 into BALANCE2

2.2) add $\$ 20$ to BALANCE2

2.3) write BALANCE2 as value of account 123

occurred must be aborted.

Other methods have been developed which remove the strict serializability conditions to allow for the sharing of partial results as is appropriate in environments such as group design or group software development. As well as those mentioned above, there have been many other developments which extend the simple transaction model for various aims, either general or specifically directed at certain environments.

\section{Motivations and Goals}

This project came about as a result of the combining of expertise in research from the fields of Persistent Systems and Database Systems. It was inspired by the current state of "multi-user support" in Persistent Systems 
research and a desire to help develop persistent systems as a practical alternative for "real world" problem solving.

Current work in persistent systems has concentrated mainly on the development of single user systems. These systems have concentrated a great deal of effort into the implementation of the persistent object support and language issues. Napier [2][6], for example. has provided a powerful environment for persistence, with a very advanced programming language. The language in the Napier system provides strong support for abstract data types, a powerful type system which supports first order procedures and polymorphism. Such systems have not, however, concentrated nearly as much effort on clean support for concurrency control or resilience. Most systems have been developed as single user environments with very simplistic transactional semantics and resilience based on simple checkpointing.

Persistent environments provide a data model which combines ease of access to long term data, which one normally finds only in database systems, with the expressive power of complex programming languages. As such they would seem to be a very appropriate alternative to database systems for many problems. There are, however, several shortcomings in the systems which have been developed to date which must be addressed if persistent systems are to become viable alternatives to database technology for "real world" problem domains. These shortcomings include (but are not limited to): efficient handling of bulk data types (such as large data tables), general efficiency and performance considerations, multi-user support and resilience considerations. The intent of this project is to address the issues of concurrency control and resilience with a main focus on concurrency control.

The goal of this project is to develop strong and flexible support for concurrency control in persistent systems. This goal is to be achieved by isolating primitive operations which must be supported at the lowest level of a persistent store to allow for the implementation of a variety of concurrency control mechanisms in higher layers. Persistent systems are powerful tools with applicability to many varied domains. These domains will require different forms of concurrency control ranging from no system provided protection through traditional transactional semantics to the more advanced concurrency control methodologies for supporting group work, long transactions and interactive work. Paralleling this need for concurrency support, is the need for resilience support which is appropriate to the forms of concurrency control which are supported.

It is intended that the support for concurrency control be implemented as cleanly and flexibly as possible. To satisfy this goal the concurrency support must be designed to avoid wherever possible making entrenchments in the lowest level of the store. The desire is to provide the means by which concurrency control can be supported while placing as little restriction as possible on the nature of the concurrency methods which may be constructed.

\section{The Experimental System}

An experimental persistent data store is being developed to aid in the investigation of concurrency control for this project, and to provide a testbed for the demonstration of the feasibility of the results of the project. The following paragraphs give a brief overview of this system in its current form as at the writing of this paper.

As this project is primarily involved with isolating and assessing primitives for concurrency control it was decided that adequate analysis of primitives could be achieved with a test system constructed on top of an accessible conventional operating system, such as UNIX, rather than embedding the primitives within the system. It was also decided that support for distribution should be included in the earliest and lowest level designs for this test system, rather than attempting to provide distribution support at a later stage. With these two decisions in mind the test system is currently implemented as a client-server architecture with a multi-threaded server accessing a centralised data store.

The centralised data store supports a pool of objects identified by unique object identifiers (OIDs) rather than direct addresses. The choice of object identifiers was made to allow for flexibility and allow for a concept of "Identity" which would simplify reasoning about interaction between concurrency control, resilience, garbage collection, memory management etc. At the lowest level the objects in the pool consist of a collection of uninterpreted data bytes and a number of references to other objects.

The interaction between the client and server is by way of requests from the client for the server to execute certain operations. The valid operations include creation of objects, reading and writing of objects data or references and operations to change the size of objects while maintaining the same OID. A small set of primitive operations are currently being added to the system to support concurrency control. These will be tested by implementing a number of concurrency control systems on the store, and the experience gained will be used to assess and modify the set of primitives.

The test system will provide a means of assessing the value and expressiveness of different sets of primitive with the intent that, when a final optimal set of primitives 
is discovered, the primitives would be embedded within the kernel along side support for persistence.

\section{Conclusion}

This project is designed to address one of the areas of persistent system development which has not received adequate investigation to date. It is hoped that this project will provide insights into the concurrency control requirements of persistent systems, which will expand the set of problem domains addressable by persistent systems and help to move them another step towards being viable alternatives for "real word" problem solving.

\section{References}

[1] Atkinson, M., Morrison, R. "Persistent System Architectures" in Persistent Object Systems Proceedings of the Third International Workshop, Newcastle, N.S.W., Australia 1989.

[2] Brown, A. L, Dearl, A., Morrison, R., Munro, D. and Rosenberg, J. "A Layered Persistent Architecture for Napier88", Proceedings of the International Workshop on Computer Architectures to Support Security and Persistence of Information, (ed J. Rosenberg and J.L.Keedy), Springer-Verlag and British Computer Society, pp. 155-172, 1990.

[3] Chase, J. S., Levy, H. M., Baker-Harvey, M. and Lazowska, E. D. "Opal: A Single Address Space System for 64Bit Architectures", Third IEEE Workshop on Workstation Operating Systems, IEEE, 1992.

[4] Dearle, A., Di Bona, R., Farrow, J. M., Henskens, F. A., Lindstrvm , A., Rosenberg, J. and Vaughan, F. "Grasshopper - A Persistent Operating System for Conventional Hardware", Proceedings, Second International Workshop on Object Orientation in Operating Systems, Dourdan, France, (ed L.-F. Cabrera and E. Jul), IEEE, pp. 81-86, 1992.

[5] Gray, J., Reuter, A., Transaction Processing: Concepts and Techniques, Morgan Kaufmann, 1993.

[6] Morrison, R., Brown, A.L, Connor, R. \& Dearle, A. The Napier 88 reference manual, PRR-77, University of St. Andrews, Scotland 1989.

[7] Rosenberg, J. "The MONADS Architecture - A Layered View", Proceedings of the 4th International Workshop on Persistent Object Systems, Morgan-Kaufmann, 1990. 